\title{
La localización original de las estatuas del Templo Bajo de Jafra en Guiza: una propuesta
}

\author{
Francisco L. BORREGO GALLARDO
}

La localización de las estatuas de Jafra en su Templo Bajo en Guiza es desconocida, dado que fueron halladas en una favissa de ese edificio. Su posible localización ha sido estudiada, entre otros, por V. Vikentiev y, más recientemente, M. Seidel. A pesar de algunos de sus aciertos, la metodología general de la que nacen dichas propuestas parece poco apropiada y alejada de los atributos internos de cada estatua. En este trabajo se realiza una crítica de esos presupuestos y se emite una propuesta alternativa para la localización de las esculturas. Para ello se tendrán en cuenta no sólo el espacio arquitectónico, sino también distintos elementos en el análisis de cada estatua que permitan entrever su localización original: cualidades físicas, contenido de las inscripciones, sentido de lectura de las mismas, atributos iconográficos de los nombres reales, presencia y función de otros personajes (diosas, halcones) o la distribución de los componentes del $z m 3-t 3 w j$ en los paneles laterales de los tronos.

\section{The original placement of the statues in the Lower Temple of Khafre in Giza: a proposal}

The original location of Khafre's statues from his Lower Temple at Giza is unknown, given that they were found in a favissa in that building. Their possible location has been studied by some scholars, such as V. Vikentiev or, more recently, M. Seidel. In spite of some correct guesses, the general methodology applied does not seem very accurate and is out of line with the internal features of each sculpture. In this article, a review of these premises is made, and an alternative proposal for the location of the statues is also given. For this purpose, it is necessary to analyse not only the architectural space, but also a number of elements which enable us to suggest their original location: physical attributes, content and reading direction of the inscriptions, iconographical features of royal names, presence and function of other characters (goddesses, falcons), or the distribution of the components of the zm3-t3wj on the lateral panels of thrones.

KeY words: Khafre, Giza, statues, Pyramid complex, Lower Temple, Royal Iconography, Old Kingdom

$\mathrm{U}$ no de los principales problemas que presenta la interpretación de los complejos funerarios reales del Reino Antiguo es la dificultad para establecer el contexto donde relieves y estatuas cobran pleno sentido y significación ${ }^{1}$. Si bien los relieves parecen haber sido objeto de diferentes estudios, no ocurre lo mismo con las estatuas que albergaron estas instalaciones. El presente trabajo es una propuesta de reconstrucción de la localización original de algunas de las esculturas que fueron creadas para el Templo Bajo del rey Jafra en Guiza, en un intento por suplir una parte de esas ausencias.

En una excavación parcial de ese templo, Mariette descubrió en 1854 una favissa con nueve estatuas de este rey², la mayoría fragmentarias.

1. Resumen reciente de esta cuestión en Arnold, 1999.

2. Mariette, 1860; Borchardt, 1911: 9-19, láms. III-V (CG 9-17); 1934: 128 (CG 1245), lám. 173; PM III/1221-23.

Fecha de recepción: 15 de enero de 2007

Fecha de admisión: 15 de mayo de 2007 
Casi medio siglo después, Hölscher ${ }^{3}$ publicó los resultados de la expedición von Sieglin de 1909-1910, poniendo al descubierto la planta del edificio ${ }^{4}$ (fig. 1). Éste se abre por su fachada oriental mediante dos portales con sendos nichos elevados, que convergen en un vestíbulo alargado; en él fue excavada la favissa. Desde el vestíbulo se accede, en dirección oeste, a una sala de culto hipóstila, con planta en forma de $\mathrm{T}$ invertida, donde fueron practicadas hasta un total de veintitrés oquedades rectangulares en el suelo.

La función de éstas y de los nichos de las entradas como receptores de estatuas queda clara por la existencia misma de la favissa, el hallazgo de Hölscher de muchos fragmentos escultóricos en el interior y perímetro del templo 5 , y el descubrimiento ocasional de otros en las inmediatas cercanías por Hassan ${ }^{6}$.

No todos los documentos escultóricos de este contexto serán estudiados en las páginas siguientes. Sólo se considerarán aquellos que, merced a su estado de conservación, cuenten con elementos susceptibles de sugerir cuál fue su localización original. De esa manera, tanto los fragmentos de estatuas hallados por la expedición von Sieglin —demasiado incompletos como para aportar datos sustanciales respecto al tema estudiado - como algunos de los hallados por $\operatorname{Hassan}^{7}$ - muy fragmentarios, y mal y parcialmente publicados - no pueden, por el mo- mento, ser objeto de este análisis. Sí es posible incluir en el corpus la mitad inferior de una estatua de Jafra encontrada por Daressy en la cercana localidad de Fustat, asignable a este templo por criterios iconográficos y epigráficos ${ }^{8}$.

Iconográficamente, las estatuas aquí estudiadas son de tres tipos. No hay lugar para describirlas en todas sus características, pero sí se referirán algunos detalles cuando sea preciso. En síntesis, puede decirse que predomina el modelo sedente, con las figuras sobre tronos tanto de

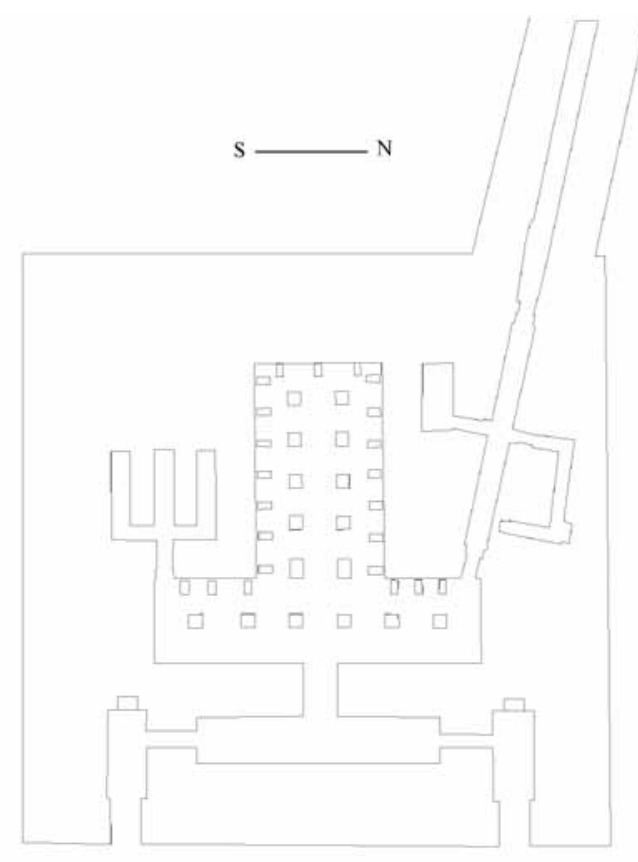

Figura 1. Planta del Templo Bajo de Jafra.

[ 90 ] 3. Hölscher, 1912

4. Sobre el edificio: Hölscher, 1912: 15-23, figs. 5-8 y 11-14, láms. II-V, VIII-XII y XVII; Maragioglio y Rinaldi, 1966: 76-88, láms. 14-16; Stadelmann, 1990: 186-189, figs. 122-123; Arnold, 1997: 51-55, fig. 14.

5. L. Borchardt, en Hölscher, 1912: $93-94$ ( $\mathrm{n}^{\mathrm{o}}$ 2-4, figs. 82-86), 96 ( $\mathrm{n}^{\mathrm{o}}$ 14, fig. 96$), 99$ ( $\mathrm{n}^{\mathrm{o}} 36$, fig. 118), 100 ( $\mathrm{n}^{\mathrm{o}} 40$, fig. 122), 101 (n ${ }^{\circ} 50$, fig. 132) y 102 (n 58 , fig. 142); Krauspe, 1997: 14-16 (cat. 2-4, láms. 2-4) y 35 (cat. 71, lám. 24.1 y 4). A este corpus preliminar habría que añadir, seguramente, algunos de los numerosos fragmentos publicados por ambos autores cuyo lugar de hallazgo dentro del complejo funerario regio es desconocido.

6. Hassan, 1943: 55, lám. XXV A; 1960a: 39 (8), lám. XIV, 82 (1), lám. XXXVII A-B (JE 72213) y 86 (4.a), lám. XLII $\mathrm{D}$ (JE 72211); PM III/1224.

7. Así, no serán tenidos en cuenta los fragmentos recogidos en Hassan, 1960a: 39 (8), lám. XIV, y 86 (4.a), lám. XLII D (JE 72211).

8. $\quad$ El Cairo RT 25.11.18.4: Daressy, 1919: 275; Stadelmann, 1998: 359-360, foto 9. 
respaldo alto como sin respaldo9. Representadas en menor número son las que presentan al rey de pie, como CG 16 y dos de las halladas por Hassan $^{10}$. Finalmente, queda mencionar que en la favissa también se encontraron los restos de una díada de Jafra con la diosa Bastet ${ }^{11}$.

\section{Propuestas anteriores}

La primera reconstrucción del espacio arquitectónico, incluyendo las estatuas, se debe a Hölscher ${ }^{12}$. En su publicación del complejo funerario de Jafra incluye una lámina con una vista ideal de la nave meridional del espacio central, de carácter ilustrativo. La siguiente propuesta, también parcial, se debe a Ricke $^{13}$, quien propuso ubicar la díada de Jafra con Bastet en el nicho del portal septentrional, postulando la posible existencia de otra díada paralela del rey con Hathor en el nicho de la puerta sur.

El primer estudioso que abordó el tema de manera general fue Vikentiev ${ }^{14}$. Comparando el Templo Bajo de Jafra con el Osireion de Sethy I en $\mathrm{Abydos}^{15}$, este autor pensó que los diecisiete huecos para estatuas de la sala principal representarían los doce meses del año más los cinco días epagómenos. Así, en el lugar de culto central, al fondo de la sala principal, ocupada por estatuas sedentes, ubica CG 14, que interpreta como una imagen del rey como Horus el Grande en el tercer día epagómeno ${ }^{16}$. La sala transversal la imagina ocupada por estatuas estantes a la manera de tríadas segmentadas: el rey con corona heráldica ${ }^{17}$ en el hueco central, rodeado hacia el exterior por Bastet al norte y Hathor al sur, y hacia el interior por Uadyet y Nejbet, respectivamente (fig. 2a). Del mismo modo, sitúa la díada con Bastet en el nicho de la puerta norte, $y$ conjetura la existencia de otra díada paralela con Hathor en el sur.

En un trabajo reciente, Seidel ${ }^{18}$ ha ensayado una reconstrucción del programa estatuario del Templo Bajo de Jafra desde criterios algo más sólidos, como las dimensiones de las estatuas, su material y algunos de sus rasgos iconográficos (fig. 2b). Este autor también piensa que habría una díada del rey con Hathor, paralela a la conservada de Jafra con Bastet, en los nichos sur y norte, respectivamente. Por otro lado, da por sentados algunos hechos para los que no existen indicios materiales sólidos. Así, presupone la existencia, para la que no hay restos, de una estatua análoga a CG 9, pero con la orientación del

9. De todas ellas: PM III/122 21-23; Stadelmann, 1998: 356-358 (iconografía de las estatuas sedentes durante la dinastía IV).

10. Hassan, 1943: 55, lám. XXV A ( $\sin ^{0}$ de inventario); Hassan, 1960a: 82, lám. XXXVII A-B; Stadelmann, 1998: 355 , foto 2 (JE 72213).

11. CG 11 + 1245: Borchardt, 1911: 11-12, lám. 3; Borchardt, 1934: 128, lám. 173; Seidel, 1996: 17-20, lám. 3, fig. 8; Stadelmann, 1998: 358, foto 7.

12. Hölscher, 1912: lám. V.

13. Ricke, 1950: 101-102.

14. Vikentiev, 1964: 9-15 y 19-21, láms. II-VII.

15. Comparación muy artificiosa y alejada de la naturaleza de la arquitectura y del programa simbólico de ambos lugares, como lo prueba la nula repercusión del trabajo de Vikentiev en autores posteriores.

16. Interpretación poco probable, no sólo porque este dios no se documenta hasta el Reino Medio, sino porque además, según las versiones conocidas, esa divinidad nació en el segundo día epagómeno: Leitz, 2002: 249-251.

17. Bajo la calificación «corona heráldica» agrupo a la $h \underline{h} t$ t dšrt y shmtj, no sólo porque cuentan con una clara vinculación territorial con las dos mitades del Doble País, sino también por su fuerte carácter emblemático, lo que la hace preferible a otras denominaciones, como la germana Landeskrone.

18. Seidel, 1996: 20-24, figs. 9-11. 
a

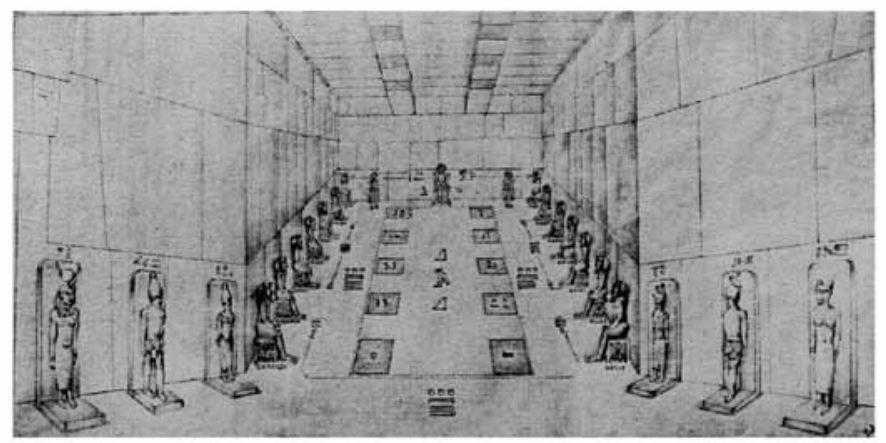

b
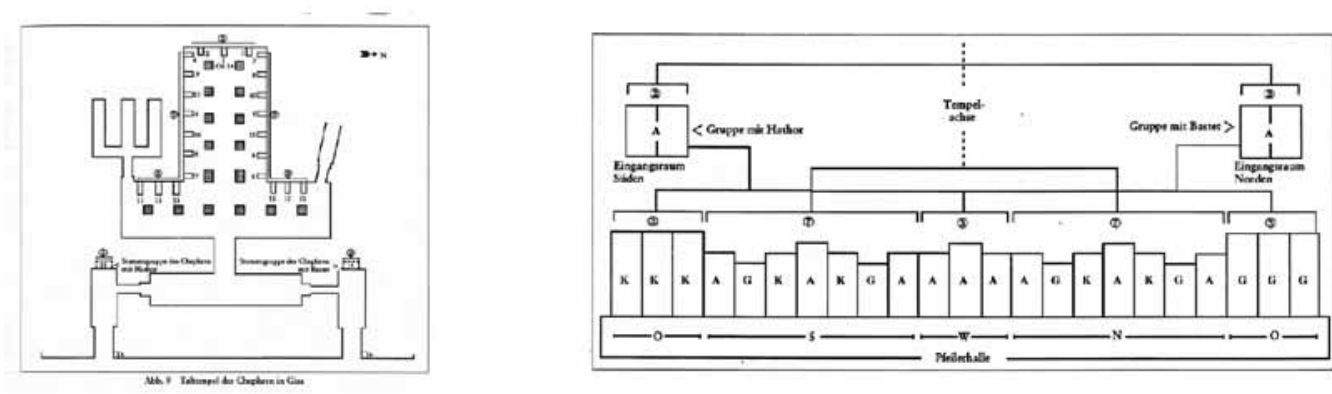

Figura 2. Hipótesis de V. Vikentiev y M. Seidel. A partir de (a) Vikentiev, 1964: lám. IV. (b) Seidel, 1996: figs 9 y 11

halcón inversa. De igual modo, cree que CG 13 llevaría una corona heráldica, y de ahí imagina la existencia de una estatua paralela con la corona complementaria. Sitúa ambas estatuas en el centro de la pared sur y norte, respectivamente, rodeadas por esculturas como CG 10,12, 15 y 17 , ritmadas y alternadas según la piedra con la que están confeccionadas. Coloca CG 14 en el [92 ] lugar de culto central, rodeado por CG 9 y su supuesto correspondiente. Por último, ubica en la sala transversal las estatuas estantes, tomando como criterio el «contraste» con la disposición de la sala más interna, con estatuas sedentes.

Metodológicamente, ambos autores, que sólo consideran las estatuas de la favissa, otorgan mucha importancia a los datos epigráficos del templo mismo, y así ubican la díada de Jafra y Bastet en el nicho del portal septentrional ${ }^{19}$. También se muestran muy condicionados por la rareza tipológica, el buen estado de conservación y alto nivel técnico de CG 14, la célebre estatua de Jafra con Behedety ${ }^{20}$, y la toman, quizá de manera inconsciente, como la escultura más importante del conjunto, lo que pudo no haber sido así para el egipcio del Reino Antiguo. Ambas reconstrucciones, además, parten de ciertos supuestos no comprobables, alejados del registro material y arqueológico, que analizan de manera parcial. Por ello, se muestran articuladas en torno a esquemas de ordenación apriorísticos e ideas preconcebidas, lo que hace muy alto su

19. Ricke, 1950: 102; Vikentiev, 1964: 12-13 y 19-20, láms. VI-VII; Seidel: 1996, 19-21.

20. Vikentiev, 1964: 11; Seidel, 1996: 22 y 24 (esta opinión de Seidel es referida por Stadelmann, 1997: 67, fig. 44). 
grado de artificiosidad y anula su verosimilitud. Desde aquí querría plantear un acercamiento diferente, partiendo de los atributos internos de cada estatua, aunque esto, a diferencia de sus reconstrucciones, no me permita ubicar de modo completamente exacto algunas de las esculturas.

\section{UNA NUEVA PROPUESTA}

Ante la inexistencia de estatuas reales del Reino Antiguo halladas in situ ${ }^{21}$, el análisis debe partir de diferentes criterios internos a las propias esculturas.

\subsection{Criterios materiales}

Un primer conjunto de elementos que permite establecer criterios de localización es el del espacio que pudieron ocupar. Las veintitrés oquedades del suelo miden $110 \mathrm{~cm}$ de largo por $60 \mathrm{de}$ ancho y 10 de profundidad ${ }^{22}$, dimensiones que encajan muy bien con las de los zócalos de las estatuas $^{23}$ (tanto sedentes como estantes), que oscilan entre los aproximadamente $100 \mathrm{~cm}$ por 60 para las primeras ${ }^{24}$ y $60 \mathrm{~cm}$ por 30 para las segundas ${ }^{25}$.

Por su parte, los nichos de las puertas, de 210 $\mathrm{cm}$ de alto, por 160 de ancho y 150 de profun$\operatorname{didad}^{26}$, pudieron haber acogido muy bien una díada como CG 11, con unas dimensiones de cerca de $120 \mathrm{~cm}$ de ancho por 60 de profundidad. La forma de esta escultura, más ancha que larga, imposibilita su localización en cualquier hueco del interior del templo — más largos que anchos-, salvo los presentes en los dos nichos de las puertas de entrada.

\subsection{Criterios epigráficos}

Otros argumentos se derivan de la orientación y contenido de los epígrafes del propio templo y de las esculturas. La dirección de las inscripciones de las estatuas sigue la orientación habitual en este tipo de soportes, mostrando una preferencia por un sentido de lectura de derecha a izquierda $(\rightarrow)$ en los diferentes epígrafes presentes

21. Excepto dos estatuas de Netcheryjet de Saqqara (JE 49158 (hallada en el serdab), y los pies de un grupo aún en el "Patio del heb-sed": Seidel, 1996: 5-9), no se han hallado estatuas regias in situ en los complejos piramidales del Reino Antiguo. Las esculturas sedentes descubiertas por Reisner (1931: 110-112 (nº 18-24), láms. 47-53), en el Templo Bajo de Menkaura, pese a lo que pudiera pensarse tras un rápido vistazo a las fotografías del hallazgo, no fueron encontradas en su posición original. Como el mismo Reisner señala (ibid.: 45 y 47), fueron movidas de su sitio e instaladas, durante la segunda fase de utilización del templo, $20 \mathrm{~cm}$ por encima del nivel del suelo original. De esa manera, metodológicamente no es muy apropiado considerarlas como paralelos seguros que aporten datos relevantes para el tema aquí tratado.

22. Hölscher, 1912: 43-44, fig. 27, lám. XVII; Maragioglio y Rinaldi, 1966: 82-83.

23. Dado su tamaño sensiblemente inferior al de las estatuas en las que se centra este estudio, parece improbable que otras esculturas cuyos fragmentos fueron hallados por Hölscher se alojaran en los huecos del suelo. Aunque algunas de ellas presenten elementos que permitan una cierta adscripción espacial, su estudio queda fuera de los límites aquí propuestos, por lo que no serán tenidas en cuenta: rey con ďrt y traje jubilar (Leipzig 1948): Hölscher, $1912: 94$ ( ${ }^{\circ}$ 4), figs. 85-86; Sourouzian, 1994: 509 (8); Krauspe, 1997: 15-16 (nº 4), lám. 4; cabecita con dšrt (Leipzig 1947): Hölscher, 1912: 93-94 (n 20), fig. 84; Krauspe, 1997: 14-15 (nº 3), lám. 3; Ziegler, en Arnold, Grzymski y Ziegler, 1999: 220 (cat. 62). Si se toman como referentes algunos contextos posteriores, mejor conocidos, como el templo de Hatshepsut en Deir el-Bahri (Tefnin, 1979: IX-X), estas estatuas inferiores en tamaño pudieron haberse localizado entre las esculturas de los huecos, entre los pilares de granito o en la terraza. Un análisis más detenido de esta cuestión será objeto de estudio por mi parte en trabajos posteriores.

24. CG 14 .

25. Medidas aproximadas de CG 16, tras la aplicación a fotografías de la pieza (como las de Stadelmann, 1998: foto 1) de algunas de las dimensiones proporcionadas por algunos autores (Borchardt, 1911: 17).

26. Medidas inferidas a partir de fotografías y dimensiones proporcionadas por Seidel, 1996: 17. 
en cada estatua ${ }^{27}$, salvo en aquellos inscritos en el lado izquierdo de cada escultura; en ese caso, dichos epígrafes siguen un sentido inverso $(\longleftarrow)$, mirando hacia el centro de la estatua. Esto se revela como un hecho especialmente importante en el caso de CG 9, pues, como ya señaló Fischer $^{28}$, la orientación - inversa a la habitualde la inscripción del respaldo del trono $(\leftarrow)$ se puede explicar por el carácter tridimensional del logograma de Horus que corona el srh esculpido en relieve en dicho respaldo. Al ser visible la figura del halcón desde el lado delantero, el ave presenta invertida esa misma orientación, que de esa manera sigue la habitual dirección de derecha a izquierda $(\rightarrow)$.

Respecto al contenido de los textos del propio templo, sólo las jambas de los portales de la fachada proporcionan datos relevantes para una localización. En el portal sur, Jafra es calificado como mry-Hwt-Hrw «amado de Hathor», mientras que al norte es mry-B3stt «amado de Bastet ${ }^{29}$. Por esta razón, como ya se ha señalado, Ricke, Vikentiev y Seidel han pensado que el nicho del portal septentrional pudo haber albergado la díada de Jafra con Bastet, lo que además es posible según el espacio disponible, el tamaño del grupo escultórico y la inexistencia de huecos más anchos que largos en el interior. Así, esta atribución parece muy segura ${ }^{30}$, y permite suponer, como ya hicieran esos autores, la existencia paralela de una díada del rey con Hathor en el nicho meridional ${ }^{31}$. Esto se ve corroborado, además de por el epígrafe y la presencia paralela de Bastet al norte, por la presencia de Hathor en otros Templos Bajos de la dinastía IV, como el de Menkaura, sucesor de Jafra ${ }^{32}$.

\subsection{Criterios iconográficos}

Un primer criterio iconográfico, de gran importancia, es la corona del halcón que preside el nombre horiano. Cuando el logograma de Horus lleva una corona heráldica, ésta es siempre la misma en todos los textos de una misma estatua, lo que muestra su coherencia como criterio válido para una ubicación espacial ${ }^{33}$. La $h \underline{d} \underline{t}$, presente en CG 10, 15, 17 y en un fragmento hallado

27. Fischer, 1983; 1986: 52-66.

28. Fischer, 1977: 29-30.

29. Hölscher, 1912: 16-17, figs. 5-6 y 8 .

30. La estatua de Bastet funcionaría, además, como el determinativo escultórico de su propio teónimo, tanto en la estatua misma como en la propia jamba de la entrada norte. En ambas inscripciones no se ha escrito determinativo alguno junto al teónimo, lo que en principio avalaría esta posibilidad.

31. Ricke, 1950: 101-102; Vikentiev, 1964: 20-21; Seidel, 1996: 19-20, lám. 4; Borrego Gallardo, en prensa. Vikentiev, 1964: 20, lám. VII, reconstruye las díadas presentando el rey ataviado con coronas heráldicas ( $d$ šrt con Bastet y hִd $t$ con Hathor, la primera leontocéfala y la segunda con cuernos liriformes y disco solar), lo que ha sido muy acertadamente criticado por Seidel, 1996: 18, quien comenta que la ausencia de pilar dorsal imposibilita que el monarca fuera portador de corona heráldica alguna, y que, por el contrario, llevara el $n m s$. La opinión del autor alemán, que considero acertada, puede verse corroborada por algunos apoyos documentales que él no cita, como las conocidas díadas de Menkaura y Hathor (Boston MFA 11.1738), Sahura y el nomo de Coptos (New York MMA 18.2.4), Nyuserra y ¿su $k a$ ? (Munich ÄS 6794), y Pepy II con Anjenesmeryra (Brooklyn BMA 39.119), y las fragmentarias de Jafra (Leipzig ¿8258? y 8233: Krauspe, 1997: 21-22 (n 26) y 22-23 ( $\left.\mathrm{n}^{\mathrm{o}} 30\right)$ ). En todas ellas el rey, tanto sedente como estante, lleva el $n m s$. Esta corona parece estar ausente de las tríadas, para las que sí se documenta el uso de coronas heráldicas (Menkaura: Boston MFA 09.200, MFA 11.3147 + Bruselas MRAH E 3074, El Cairo JE 46499, JE 40678 y JE 40679).

32. Como ponen de manifiesto la díada de Menkaura con Hathor de Boston (MFA 11.1738: Rzepka, 1998: 83-86, 88 y 90) o las triadas del mismo monarca con esa diosa (vid. n. 31; resumen con referencias de todos los ejemplares y fragmentos en Seidel, 1996: 25-42, láms. 5-16). Igualmente, Borrego Gallardo, en prensa.

33. Paralelos arquitectónicos (no escultóricos) hallados in situ: entrada secundaria al Templo Alto de Sahura (Borchardt, 1910: 34-35, lám. 8), y columnas de la wsht del mismo lugar (ibid.: 44-45, lám. 9, figs. 44-45 y 48). 
por Hassan ${ }^{34}$, permite situarlas en la mitad sur del templo. Por su parte, la estatua de Fustat contiene el nombre de Horus tocado con la $s h m t t^{35}$, lo que la emplazaría en uno de los tres nichos del fondo de la sala, o bien, teniendo en cuenta el valor múltiple de esta corona ${ }^{36}$, en cualquiera de ambas mitades, con lo que son necesarios otros criterios que permitan situarla en un lugar concreto.

Así, también son importantes los elementos iconográficos singulares dentro del conjunto iconográfico de las estatuas del Templo Bajo de este rey, que en ciertos casos permiten una localización fiable y más o menos precisa. Dada la ausencia de coronas heráldicas conservadas en las estatuas del Templo Bajo ${ }^{37}$ y la preeminencia del $n m s$ entre las figuras sedentes ${ }^{38}$, ha de prestarse atención a otros elementos que, por su rareza o excepcionalidad dentro del conjunto, permitan la localización destacada o el énfasis que se ha puesto en algunas esculturas. Tales atributos pueden ser una corona que no sea ni heráldica ni el nms (CG 13) ${ }^{39}$, un trono de res- paldo alto de leonas (CG 9, 13 y 14) ${ }^{40}$, la barba divina (CG 14), un faldellín distinto de la $\breve{s} \underline{\underline{d}} w t$ (CG 13) o la presencia de un segundo personaje, como los dioses halcones Horus en CG 9 y Behedety en CG 14.

Además, se ha de considerar que algunas estatuas se destacan del resto mediante un énfasis en la frontalidad, realizado de diferentes maneras. En primer lugar, en la parte trasera de los tronos de CG 9, 13 y 14 no se ha esculpido motivo alguno, a excepción del srh con el nombre horiano del rey en CG 9, lo que acentúa la importancia del plano anterior, frontal, de la estatua.

En segundo lugar, en CG 9 el halcón que funciona como logograma para Horus y el calificativo horiano que lo sigue presentan una orientación hacia la izquierda $(\longleftarrow)$, inversa a la habitual. Como se dijo, esta anomalía es explicada por Fischer, quien dice que el halcón, visible desde el lado frontal de la estatua, sí sigue la usual orientación hacia la derecha cuando es visto frontalmente ${ }^{41}$, cobrando plena significación desde ese

34. Hassan, 1943: 55, lám. XXVA.

35. Daressy, 1919: 275; Stadelmann, 1998: foto 9a.

36. Laboury, 1998: 82, 404 y n. 1025.

37. Sí se encontraron restos con coronas heráldicas en el Templo Alto (Krauspe, 1997: 19-20 (nº 19-20), láms. 9.2-3 (hd $\underline{d}$ ), 34-35 ( $\mathrm{n}^{\circ}$ 69-70), láms. 23.3-4 (dšrt)), y otros en zonas sin precisar (ibid.: 17 ( $\left.\mathrm{n}^{\circ} 10\right)$, lám. 6.4).

38. CG 9, 10, 12, 14, 15 y 17. Acerca del nms y sus características iconográficas en esculturas de la dinastía IV, recientemente: Stadelmann, 1998: 361-363, figs. 1-8, 11 y 13.

39. El estado fragmentario de la escultura impide establecer con seguridad con qué corona pudo haber sido tocado el monarca. Dada la ausencia de un apoyo elevado sobre el respaldo del trono (lo que descarta el uso de coronas heráldicas) y de las «aletas» del $n m s$ sobre el pecho de la estatua, los candidatos más probables son la $h 3 t /{ }^{\circ} f n t$ (un ejemplo de la dinastía IV, aunque retocado, procede del reinado de Menkaura (colección particular): Grimm, 2005: passim, esp. 19-25 y 30-32 ( $\S 2-3$ y 5) y láms. VII-XII y XIV; el uso de esta corona ya se documenta anteriormente, en relieve, con Jufu: Lauer, 1949: 111 y 114-115, lám. II; Hassan, 1960b: 23-24, lám. VIB) o la peluca jbs (Stadelmann, 1998: 365-366), tanto con la diadema š̌d (Berlín SMPK ÄM 14.396, atribuida a Jufu: Russmann, 1995: passim, esp. 117-118; Stadelmann, 1997 : 76, fig. 63; 1998: 365-366; contra: Finneiser, 1998 (lo sitúa a mediados de la dinastía V)), como sin este elemento (reinado de Menkaura: Boston 09.203: Roehrig, en Arnold, Grzymski y Ziegler, 1999: 231-232 (cat. 72); Borrego Gallardo, 2004a: 9-12 (A.3); reinado de Neferefra: JE 98279: Verner, 1985: 280, lám. LVIII; JE 98171: Verner, 1985: 272-273, láms. XLV-XLVIII; Borrego Gallardo, 2004a: 13-16 (A.4) (con referencias); JE 98178: Verner, 1985: 271, lám. XLIV; Borrego Gallardo, 2004a: 17-18 (A.5) (con referencias); dinastía V: UC 14282: Roehrig, en Arnold, Grzymski y Ziegler, 1999: 263-264 (cat. 101) (con referencias); ¿Reino Antiguo?: MRAH E.7117: Tefnin, 1988: 20-21 (nº 3)).

40. Borrego Gallardo, 2004b: 289-301, figs. 3-9 y 16.

41. Fischer, 1977: 29-30. 

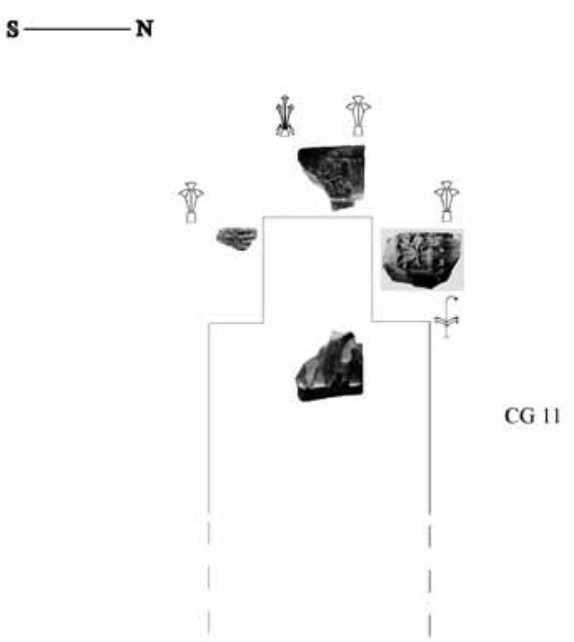

Figura 3. Localización de la díada de Jafra con Bastet $(C G 11+1245)$.

punto de vista, como en el caso de la estatuilla de Pepy I en Brooklyn ${ }^{42}$. Por su parte, la deliberada invisibilidad frontal de Behedety en CG 14, acorde a la naturaleza del dios y de su gesto $^{43}$, aboga por una localización que permitiera una plena visión frontal de la estatua, pues sólo así adquieren pleno sentido el tamaño del dios y su ocultación.

\subsection{ORIEnTACIÓN DEL $z m 3-t 3 w j$}

Otro criterio muy relevante para la ubicación de las estatuas es la disposición de las plantas que conforman el motivo del zm3-t3wj en los paneles de los tronos ${ }^{44}$. A partir de lo documentado en puertas ${ }^{45}$, altares ${ }^{46}$ y columnas ${ }^{47}$ de complejos funerarios reales de las dinastías V y VI, y de ejemplares escultóricos posteriores hallados in situ $^{48}$, el principio de ordenación es el siguiente: el papiro aparece en los lados que apuntan al norte y al oeste, mientras que el loto y el pseudolis lo hacen hacia el sur o el este. La cuestión es si esos mismos criterios pueden aplicarse a las estatuas de la dinastía IV. Gracias a la díada de Jafra con Bastet, cuya ubicación exacta se puede precisar con seguridad, se puede comprobar que, en efecto, el papiro apunta a los lados septentrional y occidental, y el loto y pseudo-lis al este y al sur (fig. 3), lo que confirma la validez de este criterio para situar las estatuas (fig. 4). En algunos casos (CG 10 y 17) no existe un encaje total de la orientación de los paneles con ninguna localización concreta, dada la disposición de los paneles laterales en quiasmo ${ }^{49}$. En esa circunstancia, el

42. Brooklyn BMA 39.120: Borrego Gallardo, 2004a: 22-25 (con referencias).

43. Ibid:: 185-222 y 287-288, esp. 203-211 y 219-222. El gesto del halcón es inteligible como protección, pero también como glorificación $(s 3 h)$ y elevación al cielo $\left(s j^{\Upsilon}\right)$, acontecidas en la $3 h t$ oriental, donde Behedety, como sol del alba (como expresan sus epítetos $s 3 b-\varsigma ̌ w t$ y $p r m$ 3ht: Borrego Gallardo, 2004c: 18-21), desarrolla su acción luminosa sin mostrarse completamente visible, en consonancia con el concepto expresado por la raíz 3h, magníficamente definida por Jansen-Winkeln, 1996: 208-213, como «eficacia invisible» (unsichtbare Wirksamkeit).

44. Resumen en Laboury, 1998: 82, con referencias.

45. Borchardt, 1910: lám. 10 (puerta entre wsht y `rrt del Templo Alto).

46. Altar de Teti en la wsht del Templo Alto: Málek, 1988: 24-28 y 30-31, figs. 1-5, láms. 3-4. También podrían añadirse los altares de Sahura (Borchardt, 1910: 48, figs. 51-54; Wartke, 1977; Málek, 1988: 30-31, fig. 5) y Nyuserra (Borchardt, 1907: 68-69, láms. 14-15; Málek, 1988: 30-31, fig. 5), asimismo encontrados en la wsht de sus templos altos respectivos, pero no hallados in situ, sino en diversos fragmentos dispersos. Merced a la distribución geográfica de sus motivos iconográficos, ambos parecen haber estado orientados de la misma manera que el de Teti.

47. Borchardt, 1910: 44-45, lám. 9, figs. 44-45 y 48 (paneles de los fustes de la wsht del Templo Alto de Sahura). El mismo principio se seguiría, a partir de este caso, en las cartelas de las columnas erigidas en el Templo Alto de Unis (Labrousse, Lauer y Leclant, 1977: 24-30, figs. 13-14 y 16-18), pero resulta más prudente no tenerlas en cuenta, al no haber sido encontradas exactamente in situ, sino caídas y fragmentadas.

48. Cf. n. 21.

49. Recurso utilizado en la gramática del templo, entre otras cosas, para reforzar la complementariedad de conceptos expresados de modo dual: Labrique, 1992: 307-310. 

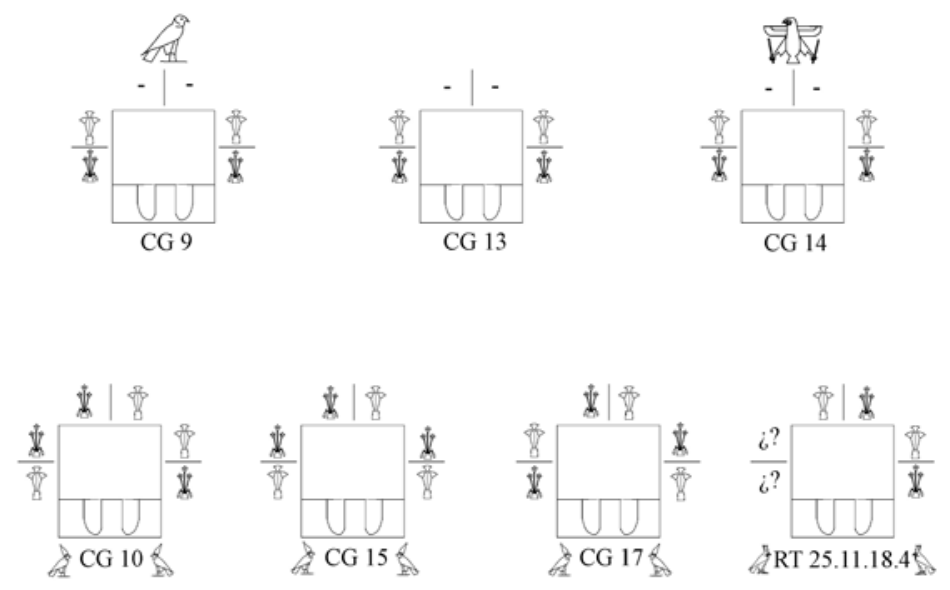

Figura 4. Distribución de personajes secundarios (dioses halcones) y elementos del zm3-t3wj en las estatuas sedentes.

panel posterior se revela como el determinante a la hora de establecer una colocación.

Conjugando entonces los criterios iconográficos, epigráficos y la orientación del $z m 3-t 3 w j$ (fig. 5), se puede señalar, al menos, en qué

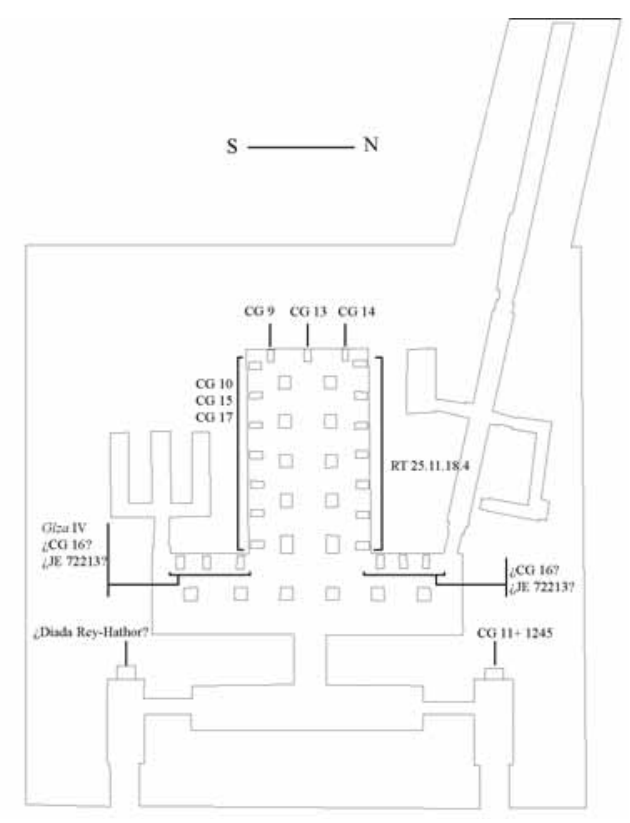

Figura 5. Propuesta de localización de las estatuas estudiadas. segmento del templo pudieron haberse ubicado las estatuas sedentes. Así, contra la pared sur de la parte interior del templo se pudieron haber emplazado CG 10, 15 y 17, y en la pared opuesta, la septentrional, la estatua hallada en Fustat. Contra la pared del fondo, la occidental, con tres huecos, se pudieron haber dispuesto CG 9, 13 y 14 .

Una cuestión que surge a continuación es si es posible averiguar la disposición interna entre estas estatuas. Los autores anteriores, sin duda influidos por el mejor estado de conservación de CG 14 y la singularidad de presentar a Behedety en su nuca, creen que esta estatua habría de ocupar el lugar central de este templo. No obstante, teniendo en cuenta los rasgos iconográficos de las tres esculturas, se aprecia que la ordenación interna de este trío de estatuas obedece a los principios de alternancia y simetría (fig. 6). El lugar central, entonces, debió de haber sido ocupado por CG 13, pues es la estatua que, a diferencia de CG 9 y CG 14, no presenta el $n m s$, ni pelucas en las leonas de su trono, no viste la $\breve{n} \underline{d} \underline{d w t} \mathrm{y}$ carece de un personaje secundario dentro de la composición, destacando la propia singularidad 
del monarca. Más difícil resulta asignar cada una de las dos estatuas restantes a una localización concreta. Sin embargo, hay un criterio que puede servir como indicio: la orientación hacia la derecha del logograma de Horus en bulto redondo de CG 9. Tomando como paralelo la orientación de las inscripciones que presentan el nombre horiano en puertas $^{50}$ o estatuas ${ }^{51}$, CG 9 pudo haber estado ubicada al sur de CG $13^{52}$, de tal manera que el halcón de Horus apuntara hacia esta última, en el centro. Por consiguiente, CG 14 estaría colocada en la oquedad más septentrional ${ }^{53}$ de las tres ${ }^{54}$.

Respecto a las estatuas con las figuras de pie, su estado de conservación, tan fragmentario, y la ausencia de $z m 3-t 3 w j$ en este tipo estatuario hace muy difícil ubicarlas. Vikentiev y Seidel las sitúan en la antesala transversal: el primero lo apunta tras comparar las dos series de tres oquedades de este lugar con las tríadas de Menkaura $^{55}$, mientras que el alemán utiliza la idea de «contraste» respecto al predominio de estatuas sedentes en la sala posterior. Ambos ar- gumentos no parecen muy sólidos, si bien, en mi opinión, sí pueden existir algunos criterios que los apoyen. Así, los huecos en esa zona son ligeramente más estrechos, lo que está en consonancia con las proporciones de las estatuas estantes. Además, el gesto de las estatuas sedentes difiere del gesto de las que están de pie en que muestran el brazo izquierdo en ademán de recibir ofrendas, es decir, como sujetos pacientes, pasivos, receptores de una acción cultual ${ }^{56}$, más propia de

\begin{tabular}{|c|c|c|}
\hline Sur & Centro & Norte \\
\hline CG 9 & CG 13 & CG 14 \\
\hline$n m s$ & no $m m s$ & $n m s$ \\
\hline $\begin{array}{c}\text { leona } \\
\text { con peluca }\end{array}$ & $\begin{array}{c}\text { leona } \\
\text { sin peluca }\end{array}$ & $\begin{array}{c}\text { leona } \\
\text { con peluca }\end{array}$ \\
\hline šndwt & no šndwt & šndwt \\
\hline $\begin{array}{c}\text { personaje: } \\
\text { Horus }\end{array}$ & sin personaje & $\begin{array}{l}\text { personaje: } \\
\text { Behedety }\end{array}$ \\
\hline
\end{tabular}

Figura 6. Ordenación interna del grupo conformado por $C G$ 9, 13 y 14.

50. Abusir: Sahura (Borchardt, 1910: láms. 8 y 10, figs. 29, 58); Saqqara: Netcheryjet: apartamentos subterráneos de la pirámide escalonada y la «tumba sur» (por ejemplo, Lauer, 1957: figs. 1, 5, 6 y 9).

51. Para el reinado de Jafra: CG 10, 15, 17; RT 25.11.18.4.

52. También presenta una naturaleza meridional la estatua que constituye el paralelo más cercano y seguro de esta estatua (reinado de Pepy I: Brooklyn BMA 39.120: $c f$. n. 42).

53. En el turno de preguntas que siguió a la presentación de esta comunicación, el Prof. Dr. J. R. Pérez-Accino —a quien desde aquí doy las gracias - tuvo la amabilidad de señalar otro argumento que podría confirmar la localización de CG 14 en este lugar. Según él, el gesto de Behedety se centra en la parte posterior de la cabeza, $m h$ 3 $(\mathrm{Wb}$. II 128,1$)$, palabra que comparte la misma raíz que $M h t$ «Delta» ( $W b$. II 125, 4-5), $M h ̣ w$ «Bajo Egipto» (Wb. 123, 12-14), etc. Así, una estatua donde está presente un gesto relacionado con la parte posterior del rey $(m h 3)$ se ubicaría en la mitad «posterior», septentrional, del templo $(M h t, M h w)$. Sobre las raíces que traducen el gesto de Behedety en CG 14: Borrego Gallardo, 2004a: 287-288.

54. A partir de las reglas de alternancia y simetría, podrían enunciarse algunas posibilidades de reconstrucción de los atributos iconográficos hoy perdidos de CG 9 y 13. La primera, sin duda, llevaba el $n m s$ en origen, y muy posiblemente la barba divina, por paralelismo con CG 14, y para no estar en desigualdad de estatus con ésta. Esto mismo puede aplicarse a CG 13, que habría de ocupar la posición central, lo que conlleva que fuera portadora de esa barba, para no quedar en desigualdad con las otras dos.

55. Vikentiev, 1964: 12-14, lám. IV, si bien él mismo admite implícitamente la ausencia de argumentos que lo apoyen, mostrando la debilidad de su planteamiento al reconocer (p. 12) que no existen posibles estatuas para esos lugares. En este sentido, tampoco tiene en cuenta CG 16 ni los dos fragmentos hallados por Hassan ( $c f$. n. 10), ya publicados cuando el autor ruso escribió su artículo.

56. Borrego Gallardo, 2004a: 137-140. 
la zona más íntima y profunda del templo que de la transversal. Pese a la posibilidad de ubicar una de las estatuas halladas por Hassan en el sector meridional de este lugar por el nombre horiano, no es posible por el momento, desde mi punto de vista, adscribirla a una de las tres plazas en particular. Más impreciso aún es el caso de CG 16 y JE 72213, cuyo estado fragmentario impide cualquier adscripción a una u otra mitad.

\section{Conclusiones}

A lo largo de este estudio se ha podido constatar que es posible una aproximación al contexto original de las estatuas mediante el análisis de algunos elementos que están en armonía con la ubicación original de cada una de ellas: dimensiones, inscripciones, atributos iconográficos y la disposición de las plantas del $z m 3-t 3 w j$. Con ello, se puede dar ya un primer paso - establecer un contexto y una ordenación de las estatuasque permita realizar lo que en realidad es mi objetivo de análisis: el estudio holístico de estas esculturas a través del método semiológico. Así, ya puedo avanzar que, en función del gesto de las estatuas, se pueden distinguir tres ámbitos diferentes, coincidentes con los tres ámbitos arquitectónicos. Así, las díadas presentan al rey como un sujeto paciente, pasivo, esto es, dativo, receptor de la acción de la diosa. Todo lo contrario sucede en la sala transversal sur-norte, donde el rey, estante, se muestra activo y con iniciativa. En la sala más interna, en cambio, el gesto de recepción de ofrendas y la presencia de un segundo personaje en algunas de ellas hace que el carácter del rey vuelva a ser pasivo, estático, dativo. El estudio de esta disposición y de sus implicaciones y significados será objeto de estudio por mi parte en trabajos sucesivos. 


\section{Bibliografía}

ARnold, D.

1997 Royal cult complexes of the Old and Middle Kingdoms, en: Shafer, B. E. (ed.): Temples of Ancient Egypt, Cornell: 31-85.

1999 Les relations entre la statuaire et l'architecture, en: Arnold, Do., Grzymski, K. y Ziegler, Ch. (comps.) (1999): L'art égyptien au temps des pyramides, Paris: 64-69.

Arnold, Do., Grzymski, K. y Ziegler, CH. (comps.)

1999 L'art égyptien au temps des pyramides. Paris.

BORCHARDT, L.

1907 Das Grabdenkmal des Königs Ne-user$r e^{r}$. Leipzig.

1910 Das Grabdenkmal des Königs Ś'aß̧̣u-re`. Band I: Der Bau. Leipzig.

1911 Statuen und Statuetten von Königen und Privatleuten im Museum von Kairo. Nr. 1-1294. Teil 1. Text und Tafeln zu Nr. 1-380. Berlin.

1934 Statuen und Statuetten von Königen und Privatleuten im Museum von Kairo. Nr. 1-1294. Teil 4. Text und Tafeln zu Nr. 9511294. Berlin.

Borrego Gallardo, F. L.

2004a Estatuas con halcones a la espalda del rey durante el Reino Antiguo. Estudio semiológico e histórico. Trabajo de In[100 ] vestigación del D.E.A. Inédito. Madrid, UAM.

2004b Los tronos de bia en los Textos de las Pirámides y las estatuas de Jafra, en Córdoba, J. $\mathrm{M}^{\mathrm{a}}$ y del Cerro, $\mathrm{M}^{\mathrm{a}} \mathrm{C}$. (eds.): L'archeologia ritrovata. Omaggio a Paolo Matthiae per il suo sessantacinquesimo aniversario - La Arqueología reencontrada. Homenaje a Paolo Matthiae en su sexagésimo quinto aniversario $I$, (Isimu 7), Madrid: 277-312. 2004c Remarques sur le dieu Béhédety dans l'Ancien Empire, TdE 3: 7-40.

en prensa Hathor y Bastet en los complejos funerarios reales de la dinastía IV, en: Córdoba Zoilo, J. M ${ }^{\mathrm{a}}$ y Sevilla Cueva, C. (eds.): En la historia, la excavación y el descifre. Una aproximación al presente de la investigación española en Oriente Próximo y Egipto, (Isimu II Series: Acta et Symposia 5), Madrid.

Daressy, G.

1919 Antiquités trouvées à Fostat, $A S A E$ 18: 275-278.

FinNEISER, K.

1998 Beobachtungen zu einem Königskopf des Alten Reiches, GM 163: 53-70.

Fischer, H. G.

1977 The Orientation of Hieroglyphs. Part I. Reversals. New York.

1983 Rechts und Links, $L \ddot{A} \mathrm{~V}:$ 187-191.

1986 L'écriture et l'art de l'Égypte ancienne. Quatre leçons sur la paléographie et l'épigraphie pharaoniques. Paris.

Grimm, A.

2005 Imagines Aegypti - Varia Ikonologica. Ein Mykerinos-Bildnis in Privatbesitz, Z̈̈S 132: 12-34.

Hassan, S.

1943 Excavations at Gîza. Vol. IV. 1932-1933. Cairo.

1960a Excavations at Gîza. Vol. IX. Season. 1936-37-38. The Mastabas of the Eighth Season and Their Description. Cairo.

1960b Excavations at Gîza. Vol. X. Season 1938-39. The Great Pyramid of Khufu and its Mortuary Chapel. With Names and Titles of Vols. I-X of the Excavations at Gîza. Cairo. 
HÖLSCHER, U.

1912 Das Grabdenkmal des Königs Chephren. Leipzig.

JANSEN-WINKELN, K.

1996 „Horizont“ und „Verklärtheit": Zur Bedeutung der Wurzel 3h, SAK 23: 201-215.

KRAUSPE, R.

1997 Katalog Ägyptischer Sammlungen in Leipzig. Band 1. Statuen und Statuetten. Mainz am Rhein.

LABOURY, D.

1998 La statuaire de Thoutmosis III. Essai d'interprétation dans son contexte historique. (AgLeod 5). Liège.

LABRIQUe, F.

1992 Stylistique et théologie à Edfou. Le rituel de l'offrande de la campagne: étude de la composition. (OLA 51). Leuven.

Labrousse, A.; Lauer, J.-PH.; Leclant, J.

1977 Le temple haut du complexe funéraire $d u$ roi Ounas. (BdE 73). Le Caire.

LAUER, J.-PH.

1949 Note complémentaire sur le temple funéraire de Khéops, $A S A E$ 49: 111-123.

1957 Remarques sur les stèles fausses-portes de l'Horus Neteri-khet (Zoser) à Saqqarah, MonPiot 49: 1-15.

LeITZ, C.

2002 Lexikon der ägyptischen Götter und Götterbezeichnungen. Band Vh-h. (OLA 114). Leuven.

MÁleK, J.

1988 The altar in the pillared court of Teti's Pyramid-temple at Saqqara, en: Baines, J., James, T. G. H., Leahy, A. y Shore, A. F. (eds.): Pyramid Studies and Other Essays Presented to I. E. S. Edwards, (EES Occasional Publications 7), London: 23-34.
Maragioglio, V.; Rinaldi, C.

1966 L'architettura delle piramidi menfite. Parte $V$. Le piramidi di Zedefrâ e di Chefren. Rapallo.

Mariette, A.

1860 Lettre de M. Mariette, Revue Archéologique 2: $16-22$.

REISNER, G. A.

1931 Mycerinus. The Temples of the Third Pyramid at Giza. Cambridge (Massachusetts).

Ricke, $\mathrm{H}$.

1950 Bemerkungen zur ägyptischen Baukunst des Alten Reichs II, $B \ddot{A B A} 5$ : 1-128.

Russmann, E.

1995 Two Heads of the Early Fourth Dynasty, en: Stadelmann, R. y Sourouzian, H. (eds.): Kunst des Alten Reiches. Symposium im Deutschen Archäologischen Institut Kairo am 29. und 30. Oktober 1991, (SDAIK 28), Mainz: 111-118.

RZEPKA, S.

1998 Some Remarks on Two Mycerinus Group Statues, GM 166: 77-90.

SEIDEL, $\mathrm{M}$.

1996 Die königlichen Statuengruppen. Band I. Die Denkmäler vom Alten Reich bis zum Ende der 18. Dynastie. (HÄB 42). Hildesheim.

SOUROUZIAN, H.

1994 Inventaire iconographique des statues en manteau jubilaire de l'Époque thinite jusqu'à leur disparition sous Amenhotep III, en: Hommages à Jean Leclant, (BdE 106/1), Le Caire: I, 499-530.

STADELMANN, R.

1990 Die großen Pyramiden von Giza. Graz.

1997 Tumbas reales de la era de las pirámides, en: Schulz, R. y Seidel, M. (eds.): Egipto. El mundo de los faraones, Colonia: 46-77. 
1998 Formale Kriterien zur Datierung der königlichen Plastik der 4.Dynastie, en: Grimal, N. (ed.): Les critères de datation stylistiques à l'Ancien Empire, (BdE 120), Le Caire: 353-387.

TEFnin, R.

1979 La statuaire d'Hatshepsout. Portrait royal et politique sous la 18 e Dynastie. (MonAeg 4). Bruxelles.

1988 Statues et statuettes de l'Ancienne Égypte. Bruxelles.
VERNER, M.

1985 Les sculptures de Rêneferef découvertes à Abousir, BIFAO 85: 267-280.

ViKenTIEV, V.

1964 Aspects symboliques du monument de Chephren comparé à celui de Seti ${ }^{\text {Ier }}$ à Abydos et à l'édifice de Saqqarah-Sud, BIE 39: 7-33.

WARTKE, R.

1977 Zum Alabaster-Altar des Königs Sahu-Rē, ZÄS 104: 145-156. 


\section{Trabajos de Egiptología Papers on Ancient Egypt}

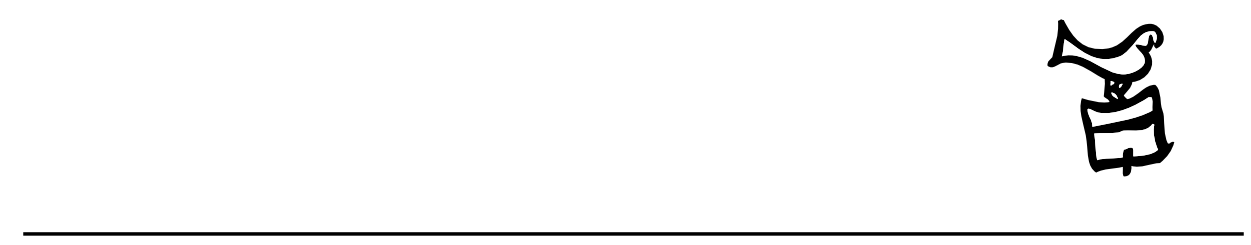

Número 5/1 2009 


\section{Actas \\ III Congreso Ibérico de Egiptología III Congresso Ibérico de Egiptologia}

Editores

Miguel Ángel Molinero Polo Covadonga Sevilla Cueva 


\title{
Editor
}

Miguel Ángel Molinero Polo

Universidad de La Laguna

\section{Consejo Editorial}

\author{
Antonio Pérez Largacha \\ Universidad de Castilla-La Mancha \\ José-R. Pérez-Accino \\ Birkbeck, Universidad de Londres \\ Covadonga Sevilla Cueva \\ Universidad Autónoma de Madrid
}

\section{Comité Científico}

Josep Cervelló i Autuori

Universitat Autònoma de Barcelona

$\mathrm{M}^{\mathrm{a}}$ José lópez Grande

Universidad Autónoma de Madrid

Josep Padró i Parcerisa

Universitat de Barcelona

$\mathrm{M}^{\mathrm{a}}$ Carmen Pérez Die

Museo Arqueológico Nacional, Madrid

Ester Pons Mellado

Museo Arqueológico Nacional, Madrid

José M. Serrano Delgado

Universidad de Sevilla

\section{Colaboradores Editoriales}

Linda Steynor

English editorial assistant

Hervé Mouriacoux

Assistant éditorial pour la langue française 
Trabajos de Egiptología está producida por Isfet. Egiptología e Historia c/ Blanco $1,2^{\circ}$

38400 Puerto de la Cruz

Tenerife-Islas Canarias

España

Maquetación: Proyecto Limón

(C) Autores de los artículos aparecidos

y Consejo Editorial de Trabajos de Egiptología - Papers on ancient Egypt

Depósito Legal: TF-2302-2009

ISSN: $1695-4750$

Imprime: Gráfica Los Majuelos, S.L.L.

imprenta@graficaslosmajuelos.com

Tfno.: 922311455 


\section{Comité Científico \\ III Congreso Ibérico de Egiptología III Congresso Ibérico de Egiptologia}

Miguel Á. Molinero Polo

Universidad de La Laguna

Presidente del Comité Organizador del III Congreso Ibérico de Egiptología

Miembro del Comité Organizador del I Encuentro de Egiptología

Josep Cervelló Autuori

Universitat Autònoma de Barcelona

Presidente del Comité Organizador del II Congreso Ibérico de Egiptologia

José Manuel Galán Allué

Consejo Superior de Investigaciones Cientificas

Director del Proyecto Djehuty, Luxor, Egipto

$\mathrm{M}^{\mathrm{a}}$ Helena Trindade Lopes

Universidad de Lisboa

Directora de la Misión Arqueológica Portuguesa en Menfis

Josep Padró i Parcerisa

Universitat de Barcelona

Director de la Misión Arqueológica de Oxirrinco

Antonio Pérez Largacha

Universidad de Castilla - La Mancha

Miembro del Comité Organizador del I Encuentro de Egiptología

José Ramón Pérez-Accino

Birkbeck College, University of London

Miembro del Comité Organizador del I Encuentro de Egiptología

$\mathrm{M}^{\mathrm{a}}$. Carmen Pérez Díe

Museo Arqueológico Nacional

Directora de la Misión Arqueológica Española en Heracleópolis Magna, Egipto

Covadonga Sevilla Cueva

Universidad Autónoma de Madrid

Miembro del Comité Organizador del I Encuentro de Egiptología 